
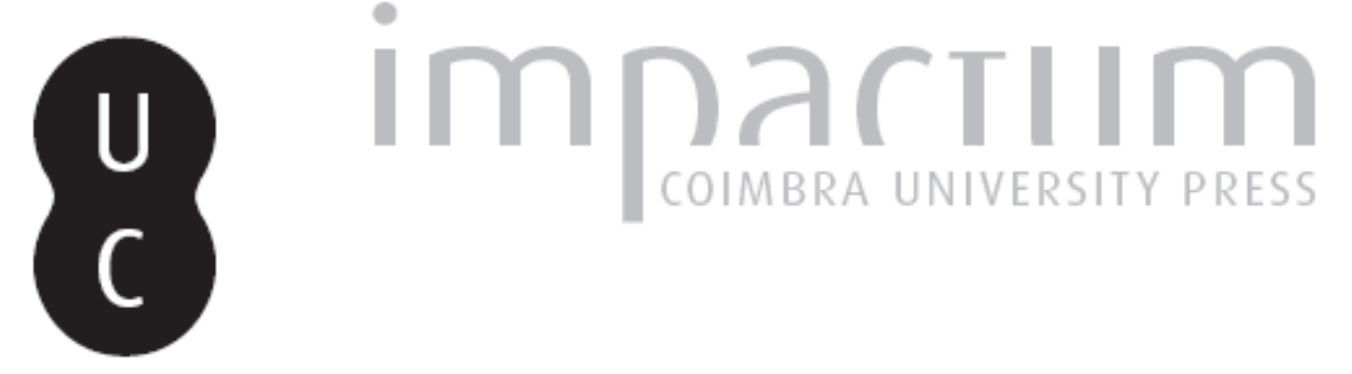

\title{
Compte-rendu de: Francesco Fronterotta,. La teoria platonica delle idee e la partecipazione delle cose empiriche. Dai dialoghi giovanili al Parmenide
}

Autor(es): $\quad$ Morel, Pierre-Marie

Publicado por: Imprensa da Universidade de Coimbra

URL

persistente:

URI:http://hdl.handle.net/10316.2/42261

DOI:

DOI:https://doi.org/10.14195/2183-4105_3_3

Accessed : $\quad$ 26-Apr-2023 10:26:48

A navegação consulta e descarregamento dos títulos inseridos nas Bibliotecas Digitais UC Digitalis, UC Pombalina e UC Impactum, pressupõem a aceitação plena e sem reservas dos Termos e Condições de Uso destas Bibliotecas Digitais, disponíveis em https://digitalis.uc.pt/pt-pt/termos.

Conforme exposto nos referidos Termos e Condições de Uso, o descarregamento de títulos de acesso restrito requer uma licença válida de autorização devendo o utilizador aceder ao(s) documento(s) a partir de um endereço de IP da instituição detentora da supramencionada licença.

Ao utilizador é apenas permitido o descarregamento para uso pessoal, pelo que o emprego do(s) título(s) descarregado(s) para outro fim, designadamente comercial, carece de autorização do respetivo autor ou editor da obra.

Na medida em que todas as obras da UC Digitalis se encontram protegidas pelo Código do Direito de Autor e Direitos Conexos e demais legislação aplicável, toda a cópia, parcial ou total, deste documento, nos casos em que é legalmente admitida, deverá conter ou fazer-se acompanhar por este aviso.

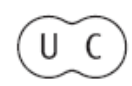




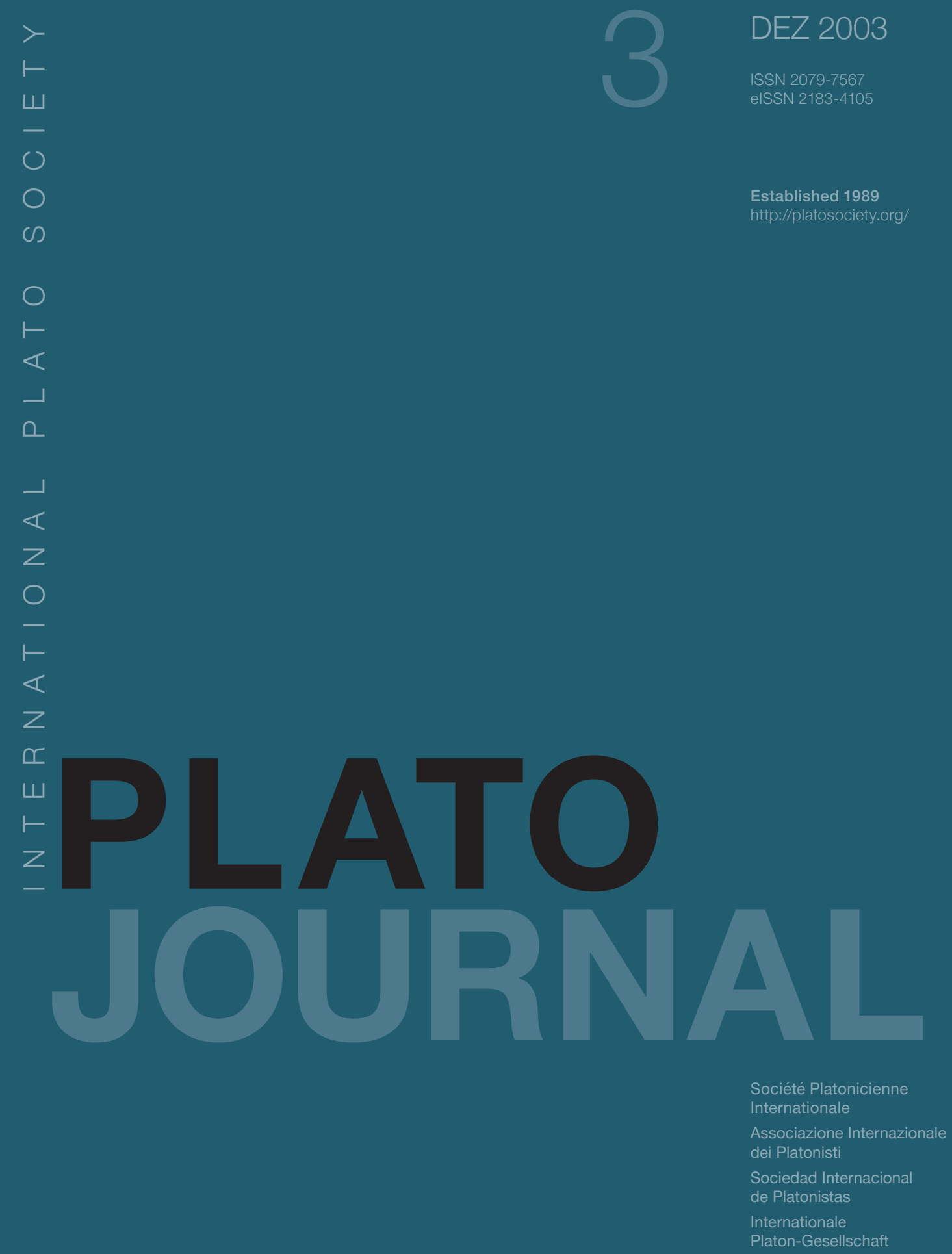




\section{Compte-rendu de : \\ Francesco}

Fronterotta, $M E \Theta E$

$X I \Sigma$. La teoria

platonica delle idee $e$

la partecipazione

delle cose empiriche.

Dai dialoghi

giovanili al

Parmenide

MOREL, Pierre-Marie, in 3. Plato 3

(2003) , [En ligne] , January 2003

Dans ce volumineux ouvrage, F.F. procède à une étude d'ensemble de la doctrine des idées, qu'il reconstruit pas à pas. La première partie (" Epistemologia e ontologia : la formulazione della teoria delle idee ") étudie la référence aux idées dans les dialogues de jeunesse, puis l'ontologie et l'épistémologie des dialogues intermédiaires et de la maturité. La seconde partie (" La teoria delle idee e il dilemma della partecipazione nel Parmenide ") examine la façon dont le problème de la participation est posé, non seulement dans la première section, mais aussi dans la seconde section du Parménide. Trois appendices complètent l'analyse en ouvrant des perspectives vers les derniers dialogues - le Sophiste (appendice I) ; le Philèbe et le Timée (appendice II) - et en introduisant à la critique aristotélicienne de la doctrine des idées (appendice III). Suivent une bibliographie de 25 pages et les indices des auteurs modernes et des passages cités.

F.F. ne croit pas que les premiers dialogues aient ignoré les formes intelligibles et sa reconstruction du développement progressif de la doctrine laisse peu de place aux hypothèses les plus radicales des spéculations de type génétique. Il rappelle tout d'abord que nous ne disposons d'aucune indication sûre concernant la chronologie des dialogues. On se contentera donc d'admettre l'appartenance générique de tel ou tel dialogue à l'une des trois périodes généralement identifiées (jeunesse, maturité, vieillesse). F.F. n'élimine donc pas par principe toute évolution, mais il privilégie le point de vue systématique par rapport à l'axe chronologique.

Il affine dans un deuxième temps sa conception de l'ensemble, en distinguant, pour les dialogues de jeunesse, les différentes étapes d'un même parcours qui conduirait à l'affirmation doctrinale de la théorie des idées. Ainsi, l'Hippias Majeur est à ses yeux un tournant dans la démarche définitionnelle de Platon, par rapport au Lachès et auCharmide, ce qu'il explique de la manière suivante. La question " qu'est-ce que le beau ? " reçoit dans l'Hippias Majeur une réponse qui ne se situe pas simplement sur le plan épistémologique - une bonne définition doit être logiquement cohérente avec l'ensemble des propositions que l'on soutient à propos d'un definiendum $x$ - mais aussi, et plus fondamentalement, sur le plan ontologique. La question n'est pas en effet " quelle chose est belle ? ", mais " quelle chose est le beau ? ", de sorte que l'Hippias Majeurmet l'accent sur " le nécessaire référent objectif de la définition " (" il necessario riferimento oggetivo della definizione ", p. 15). Or l'objet requis par la question doit être unique et universel et ne peut donc qu'être une forme ou une idée. Selon F.F., les caractéristiques fondamentales de l'idée seraient donc implicitement convoquées dès ce stade : immuable et toujours identique à soi, présente dans une pluralité de choses particulières et leur conférant, en vertu de cette présence, la caractéristique dont elle est l'essence (pp. 17-18). Une étape supplémentaire est franchie avec l'Euthyphron, dialogue on le sait très discuté dans la perspective de la théorie des idées. Toutefois, pour F.F., la nouveauté de celui-ci se résume principalement à l'apparition du terme paradeigma - dont il ne doute pas qu'il désigne l'idée - en 6e. C'est surtout avec leCratyle, selon lui, que l'on parvient à une position générale sur la possibilité d'attribuer aux choses particulières une essence stable, en montrant que c'est là la condition d'un usage légitime du langage (pp. 27-33). 
D'une manière générale, tout en reconnaissant sa dette à l'égard de G. Vlastos et des études qui se sont inspirées de lui, F.F. montre que l'homologia entre les interlocuteurs ne se situe pas simplement sur le plan de l'accord sémantique, ni même sur le seul plan de la connaissance de l'objet en question, mais qu'elle exige aussi un accord sur le statut ontologique du definiendum, l'existence même de $x$, comme forme intelligible (pp. 37-38). Il distingue ainsi trois niveaux d'homologia, apportant une lumière nouvelle sur un point particulièrement débattu. Grâce à lui, le problème sans cesse réactivé de la finalité de l'elenchos socratique est enfin posé en des termes clairs et systématiques.

Les dialogues de la maturité ne sont donc pas le lieu d'émergence d'une doctrine ontologique absolument inouïe, parce que la forme intelligible telle qu'ils la conçoivent opère déjà dans les dialogues antérieurs. On pourrait objecter à F.F. que si l'Hippias

Majeur permet de reconnaître la fonction causale de la forme dans un cas déterminé (le beau), il ne dit pas qu'il s'agit de la fonction causale de la forme en tant que telle, fonction valable pour toutes les occurrences (le beau, le juste, l'égal). De ce point de vue, le Phédon marque bien une rupture par rapport aux enquêtes supposées antérieures. D’une manière générale, les dialogues de jeunesse se servent des formes d'une manière encore instrumentale - pour définir, ou du moins pour essayer de définir et pour mettre à l'épreuve les définitions proposées -, et celles-ci ne sont pas encore les véritables objets de l'activité philosophique. Il y a une inflexion nette, à propos du beau, dans le Banquet, qui ne se contente pas de dire que le beau n'est aucune des choses belles particulières, mais qu'il est séparé et doit être, comme tel, l'objet du désir authentiquement philosophique. On comparera en ce sens Hippias Majeur, 289d etBanquet, 210e-212a. Comme le note lui-même F.F. en abordant les dialogues de la maturité, la discussion de leurs principaux thèmes - l'amour, le beau, la justice, l'éducation, l'état, la nature immortelle de l'âme - exige une définition préliminaire du statut ontologique de leurs objets et des modes de connaissance qui leur conviennent.

F.F. rassemble de manière claire et textuellement argumentée, à partir de la séquence République-Banquet-Phédon-Phèdre, les énoncés qui expriment les principes fondamentaux de la " théorie des idées " (pp. 43-44). Du même coup, on peut s'interroger sur la légitimiter de l'expression même de " théorie des idées ", imposée par la commodité scolaire : si l'on est obligé de collecter, comme le fait F.F., les énoncés qui sont censés la constituer, c'est qu'il n’y a pas de théorie littérale des idées. Or, si Platon n'a pas pris soin de l'exposer, c'est peut-être qu'il n'a pas cru devoir présenter en un système l'ensemble des énoncés généraux qui caractérisent la dialectique. Après tout, le dialogue qui évoque de la manière la plus systématique l'ensemble de la doctrine des formes intelligibles, à savoir le Parménide, est aussi celui qui met en cause sa cohérence de la manière la plus directe.

En réalité, F.F. répond indirectement à cette difficulté, en reléguant au second plan la question de la théorisation de la doctrine des idées et en justifiant le recours aux formes intelligibles par une nécessité épistémologique. Il choisit donc de privilégier le point de vue épistémologique par rapport au point de vue ontologique, même s'il montre dans le cours de l'argumentation qu'ils renvoient sans cesse l'un à l'autre. Il établit en tout cas que la doctrine des idées ne se fonde pas sur un acte purement dogmatique de postulation de l'être, mais sur une implication nécessaire de la théorie de la connaissance, à partir du moment où l'on pose la question suivante : que peut-on connaître et comment le connaître ? En vertu du principe de l'adéquation (adeguamento) de la connaissance à son objet, nous devons poser des objets de pensée indépendants de la pensée, mais celle-ci doit être adéquate et uniforme à la réalité objective de son contenu. Seule donc la réalité interne, immuable et universelle des idées peut fonder une connaissance universelle, immuable et éternellement vraie (voir déjà p. xviii).

L'interprétation que F.F. donne du statut de l'idée est ainsi résolument objectiviste et réaliste. Il ne s'agit donc pas, en faisant prévaloir l'exigence épistémologique, de réduire l'idée au concept ou à un état psychique. C'est dans cette perspective que doit être comprise la problématique de l'ensemble du Parménide, c'est-à-dire non seulement celle de la première section du dialogue - où le problème du statut des formes est très explicitement posé -, mais encore celle qui anime la seconde section : une idée est-elle un être séparé de tout ce qui est autre que lui, une pure substance individuelle, ou un être qui, par sa nature même, est sujet à la participation des choses sensibles ? C'est là la double originalité de cette entreprise : établir l'unité du Parménide et montrer que les idées sont originellement liées au sensible. F.F. rappelle à ce sujet ce que nous savons et que les textes disent clairement, mais il projette un éclairage nouveau, problématique, sur ce donné familier. Ainsi, par opposition à la sphère des idées, les choses sensibles sont instables, objets d'opinions incertaines et faibles, privées d'une existence déterminée et c'est ce que F.F. appelle la " radicale minorità ontologica " des choses sensibles (p. 146). Cela, nous le savons. Il invite toutefois à reconsidérer le statut des choses sensibles, au sein même de " l'ontologie bipolaire " qui est celle de Platon (p. 148) : la participation impose " l'exigence pour ainsi dire dynamique que subsiste, entre les idées et les choses, une relation réciproque (reciproca relazione) ". Ce point est particulièrement intéressant et assez subversif pour la doctrine de la participation. Si l'on a bien compris le propos de F.F., nous sommes devant la situation suivante : si le fait pour $x$ de participer à la forme $X$ signifie que $X$ est " ce par quoi " $x$ existe et a telles et telles qualités; si la relation de participation est essentielle, non seulement à $x$, mais aussi à $X$; alors, $X$ ne peut être conçu indépendamment d'un $x$ caractérisé pourtant par son " infériorité ontologique radicale ". Cette situation oblige donc à se demander si la réalité, fonctionnelle, de la participation n'altère pas le statut paradigmatique de l'idée.

Notons tout de suite que les formes ne sauraient cependant être affectées par la participation des choses sensibles, parce que la relation causale n'est pas bijective et qu'elle est pour Platon ontologiquement inégalitaire. Aussi, plutôt que de parler d'une relation entre les formes et les choses sensibles, F.F. insiste sur la variété des relations que les choses sensibles entretiennent avec les formes : non seulement la relation causale, mais aussi la présence, la ressemblance - dans le cadre de la copy-model theory, selon la formule qu'il reprend à R.E. Allen -, ainsi que l'hypothèse d'un processus dynamique d'assimilation des choses aux idées, hypothèse 
envisagée enParménide, 132d. Cette pluralité de modes de relations ne lève pas pour autant, selon F.F., l'aporie qui découle de la non-réciprocité de la relation causale (p. 216). Lacopy-model theory est d'ailleurs à ses yeux un paradigme insuffisant car, s'il réussit peut-être à justifier le résultat du rapport participatif - la production d'une image ressemblante -, il n'explique pas son actuation concrète - comment les choses sensibles en viennent à participer à l'idée. De plus, si les idées sont des causes efficientes réelles des choses sensibles, par l'intermédiaire du rapport participatif, ne devons-nous pas admettre une forme de contact et ainsi de mélange incompatible avec la thèse de la séparation ontologique (pp. 219-221)?

L'analyse du Parménide permet de dégager un certain nombre de résultats positifs. On suivra avec intérêt la façon dont F.F. prend position dans le débat sur l'auto-prédication de l'idée, c'est-à-dire sur la question de savoir comment préserver l'idée de ses prédicats, lorsque ceux-ci sont contraires à son statut ontologique d'idée - l'idée de la multiplicité est-elle elle-même multiple ? F.F. note que l'idée de justice et l'idée de multiplicité sont seulement " formellement " et non " concrètement " juste et multiple, parce qu'elles expriment " l'essence " de la justice et de la multiplicité, qui est dans chaque cas unique, et restent ainsi uniques et identiques à elles-mêmes. Les choses sensibles, quant à elles, possèdent ces prédicats " matériellement ". En effet, chaque idée singulière possède de manière exclusive la caractéristique spécifique dont elle est l'idée. L'idée de multiplicité sera donc une, en tant qu'idée, et ne sera multiple que formellement, en tant qu'idée de la multiplicité. Cette solution, ontologique et non purement logique, permet de sortir de manière assez subtile de l'aporie de la prédication et révèle en outre un aspect essentiel de la doctrine des formes : l'articulation entre le statut ontologique propre de l'idée et le fait qu'elle soit prédicable des choses sensibles (pp. 265-269).

Toutefois, si la capacité de participer qui est celle du sensible ne lui vient ni des idées ni du sensible lui-même, il paraît nécessaire de faire intervenir un agent ou une cause externe (p. 286), préoccupation que F.F. attribue à la fois au Parménide, au Philèbe et plus particulièrement au Timée. L'appendice II prolonge cette réflexion, en montrant que le démiurge du Timée représente de manière pertinente la cause de la génération des choses sensibles, auxquelles il confère la forme et la structure des paradeigmataidéaux. Il ne procède pas en effet à une creatio ex nihilo, puisqu'il doit opérer en usant d'éléments pré-existants, auxquels il doit simplement attribuer un ordre déterminé. La fonction philosophique de cette figure mythologique est donc d'être un " trait d'union ontologique entre les choses sensibles et les idées ", tout en différant de chacun de ces deux aspects du réel (p. 389). Il remplit ainsi le rôle dévolu à la cause intelligente dans le Philèbe. La chôra, de son côté, a pour fonction théorique d'expliquer comment la participation advient concrètement aux choses sensibles. Son statut obscur et équivoque n'enlève rien à la nécessité de son intervention (p. 390). Grâce à elle, en effet, la présence matérielle des déterminations intelligibles dans le sensible n'est pas celle de l'idée, mais celle des images produites sous l'impulsion du démiurge, et ne contredit pas la différence ontologique originaire. En imposant, par opposition à l'idée d'une participation comme relation concrète et objective entre deux mondes séparés, la métaphore de la fonction ontologique et cosmologique du démiurge, le Timée enlève à la participation sa dimension aporétique. Nous pourrions nous croire tirés d'affaire s'il ne restait à trancher sur la question, cruciale et préjudicielle, de l'interprétation qu'il convient de faire de l'eikos muthos que constitue le dialogue dans son ensemble. Or F.F. invite le lecteur à s'en tenir à ce constat, très explicite dans le texte de Platon, que nous ne pouvons rendre compte rationnellement de la chôra. Le Timée n'introduit donc pas une cohérence nouvelle : il dresse " un bilan rigoureux et un cadre exemplaire de l'ontologie platonicienne et de ses difficultés ", parce que le recours au muthosn'est pas tant un instrument auxiliaire de la raison qu'une " métaphore a-rationnelle (ou irrationnelle) ", qui intervient là où le discours rationnel et la philosophie elle-même atteignent leur limite irréductible (p. 395).

L'interprétation proposée se distingue donc par l'attention qu'elle porte à la complexité de la doctrine des formes. F.F. maintient dans toute sa radicalité la séparation des idées par rapport aux choses sensibles et la discontinuité ontologique qui conduit aux apories du Parménide (voir par exemple p. 78). La doctrine de la participation établit tout à la fois la séparation et la communauté entre les " deux niveaux de réalité ", qui correspondent aux deux degrés de la connaissance (voir notamment I, v : Le idee e le cose empiriche). Ce n'est finalement qu'en reconstituant la dialectique de la continuité et de la discontinuité que l'on peut saisir le sens même de la doctrine. Cette dernière apparaît comme " une tentative constante pour superposer, à la bipolarité ontologique originaire et discontinue, l'exigence dynamique de la continuité, sans toutefois qu'à aucun moment la continuité 'reconquise' puisse être comprise comme absolue et permanente " (p. 316). Cette situation conflictuelle n'est pas annulée par les médiations que constituent la participation (p. 312), l'âme et le recours au muthos (pp. 326-328). Les critiques d'Aristote, telles qu'elles sont formulées dans la Métaphysique et dans lePeri Ideôn (voir appendice III), ne sont donc pas sans fondement et l'on apprécie de voir ici un spécialiste de Platon échapper à la tentation apologétique. F.F. n'entend pas en effet défendre à toute force la cohérence du platonisme originel contre la mauvaise foi supposée du Stagirite. Platon n'en est pas moins cohérent, comme le note subtilement F.F. en conclusion (p. 329), dans la mesure où la doctrine des idées est le reflet d'une aporie objective : en vertu même du principe d'assimilation du connaissant et du connu, la doctrine est nécessairement marquée par la tension qu'elle exprime et qui n'est autre que celle du réel.

F.F. propose donc une reconstruction originale et particulièrement approfondie de la doctrine des idées, rappelant ainsi que les problèmes les plus classiques et les plus abondamment traités sont aussi, souvent, les plus intéressants. Encore faut-il avoir le courage de les poser à nouveaux frais, sans rien tenir pour acquis de ce que l'exégèse prétend avoir établi. C'est précisément ce qui est fait ici.

\section{PIERRE-MARIE MOREL}

Université de Paris I - Panthéon-Sorbonne

France 